\title{
Colonização de clareiras naturais na floresta atlântica no sudeste do Brasil
}

\author{
MARCELO TABARELLI ${ }^{1}$ e WALDIR MANTOVANI ${ }^{1}$
}

(recebido em 22/06/95; aceito em 22/10/96)

\begin{abstract}
Treefall gaps colonization in the southern Brazil coastal forest). The natural plant colonization was analyzed in 23 treefall gaps (34-256 $\left.\mathrm{m}^{2}\right)$ of two forest sites in the Atlantic coast southern Brazil. In both plots, small gaps $\left(<150 \mathrm{~m}^{2}\right)$ were more abundant, comprising the largest area covering this type of disturbance (100-66\%). Treefall gaps shown a common colonization pattern, where shade tolerant species were more abundant and frequent than shade intolerant ones. Relationships between gap area, guild composition and abundance of dominant species were not observed. The results suggest that the Atlantic forest has a reduced richness of shade intolerant species, mainly the large pioneer ones. The gap area is not the best predictor of floristic and ecological composition in treefall gaps.
\end{abstract}

RESUMO - (Colonização de clareiras naturais na floresta Atlântica no sudeste do Brasil). Em duas florestas na encosta atlântica no sudeste do Brasil, foi analisada a colonização vegetal de 23 clareiras naturais (34-256 m²). Nos dois locais de estudo, as clareiras pequenas foram as mais abundantes, cobrindo a maior parte da superfície ocupada por este tipo de perturbação (100-66\%). As clareiras apresentaram padrão comum de colonização, caracterizado pela dominância de indivíduos e espécies tolerantes à sombra, principalmente aquelas de sub-bosque. Não observou-se relação entre tamanho das clareiras, composição de guildas de regeneração e a abundância das espécies dominantes. Os resultados sugerem que a floresta atlântica possui riqueza reduzida de espécies intolerantes à sombra, principalmente as grandes pioneiras, que são pouco abundantes e pouco freqüentes. $\mathrm{O}$ tamanho não é o melhor preditor da composição florística e ecológica das clareiras.

Key words - Treefall gaps, colonization, diversity, Atlantic forest

\section{Introdução}

A abertura de clareiras naturais é considerada um mecanismo de manutenção da diversidade de árvores em florestas tropicais (Whitmore 1978, 1982, Hartshorn 1980, Brokaw 1982), exercendo pressões seletivas sobre diversos atributos da história de vida destas populações (Bazzaz \& Pickett 1980, Hubbell \& Foster 1983, Martinez-Ramos, 1985).

Constituem um sítio imprescindível para a regeneração das espécies intolerantes à sombra, as quais ocorrem em todas florestas tropicais (Berg 1978, Vazquez-Yanes \& Sada 1985, Whitmore 1990). Hartshorn (1978) estimou que $50 \%$ das árvores de La Selva, Costa Rica, dependem de clareiras para obter êxito na regeneração.

Ao contrário de Hartshorn (1978), poucos estudos têm demonstrado a dependência efetiva das espécies em relação às clareiras e estimado a contribuição de cada guilda de regeneração (Hubbel $\&$ Foster 1986) para a riqueza das florestas tropicais, as quais constituem ambientes muito variados

1. Departamento de Ecologia Geral, IB, USP, Caixa Postal 11461, 05422-970 São Paulo, SP, Brasil.
(Terborgh 1992). Whitmore (1989) sugeriu que em florestas tropicais onde predominam clareiras pequenas, espécies tolerantes à sombra seriam mais abundantes do que as intolerantes, compondo todas as fases sucessionais do mosaico florestal, inclusive a fase clareira.

Conforme Orians (1982), existem árvores especialistas nas diferentes zonas que compõem uma clareira. Existem, também, aquelas especialistas em clareiras de diferentes tamanhos (Denslow 1980). Brandani et al. (1988) analisaram a distribuição de 104 espécies de árvores em 137 zonas de clareiras em La Selva, concluindo que muitas delas ocorrem preferencialmente em determinadas zonas.

Características estruturais das clareiras, tais como tamanho, forma, origem e idade, geram condições ambientais singulares, permitindo que clareiras diferentes apresentem padrões particulares de colonização vegetal (Brokaw 1982, 1985, Denslow 1988). A partição destes sítios é um mecanismo importante na estruturação das comunidades (Denslow 1980, 1985), principalmente no que se refere à coexistência das espécies (Begon et al. 1990).

Ao contrário de Orians (1982) e Denslow (1980, 1985), Hubbell \& Foster $(1986,1987)$ questionaram a diferenciação de nicho de regeneração como 
principal mecanismo para a coexistência das árvores nas florestas tropicais. Para estes autores, as árvores compõem guildas de espécies generalistas.

Este estudo apresenta o padrão de colonização observado em clareiras naturais em duas florestas, dentro dos domínios da floresta atlântica, no sudeste do Brasil. Testa as hipóteses de que na floresta atlântica as espécies apresentam partição de nicho regenerativo em relação ao tamanho das clareiras, as quais são um sítio de regeneração imprescindível para um número elevado de espécies de árvores e arbustos. Esperava-se encontrar: (1) uma relação entre o tamanho da clareiras e suas composições de guildas; (2) espécies relacionadas aos diferentes tamanhos de clareiras e (3) riqueza e abundância elevada de espécies intolerantes à sombra.

\section{Material e métodos}

Locais - Os estudos foram realizados no Parque Estadual da Cantareira, São Paulo, SP $\left(23^{\circ} 22^{\prime}\right.$ S, $46^{\circ} 26^{\prime}$ O) e na Estação Ecológica da Juréia, Iguape, SP $\left(24^{\circ} 32^{\prime} \mathrm{S}, 47^{\circ} 14^{\prime} \mathrm{O}\right)$ (figura 1$)$.
Segundo Veloso et al. (1991), ambas as Unidades de Conservação localizam-se dentro dos domínios da floresta ombrófila densa (tabela I).

A colonização das clareiras - As clareiras naturais foram definidas conforme Brokaw (1982), incluindo-se aquelas com regeneração natural com altura superior a $2 \mathrm{~m}$. Suas áreas foram estimadas utilizando-se a fórmula de elipse, tomando-se as medidas junto a projeção no solo da copa das árvores do dossel adjacente. São consideradas pequenas, as clareiras com áreas inferiores a $150 \mathrm{~m}^{2}$ e grandes, aquelas com áreas iguais ou superiores (Brokaw 1985).

$\mathrm{Na}$ floresta na Cantareira foram analisadas seis clareiras naturais, sendo amostrados todos os indivíduos com alturas entre $0,5 \mathrm{~m}$ e $1,3 \mathrm{~m}$. Na Juréia, foram analisadas 17 clareiras amostrando-se todos os indivíduos com perímetro do caule na altura do peito (PAP) superior a $5 \mathrm{~cm}$.

As espécies encontradas foram classificadas em duas guildas de regeneração: (a) intolerantes à sombra; a qual inclui as espécies que apresentam indivíduos jovens somente em clareiras naturais (Hartshorn 1978), as pioneiras descritas por Budowski (1965) e as espécies que apresentam indivíduos jovens em clareiras e sob o dossel da floresta pioneira, denominadas grandes pioneiras (Swaine \& Whitmore 1988) e (b) tolerantes à sombra, as quais apresentam indivíduos jovens em clareiras e sob o dossel da floresta madura (Hartshorn 1978). Neste grupo encontram-se as espécies típicas de sub-bosque, que completam seus ciclos de vida no interior da floresta, e aquelas de dossel, que toleram a sombra somente nos estágios não reprodutivos.

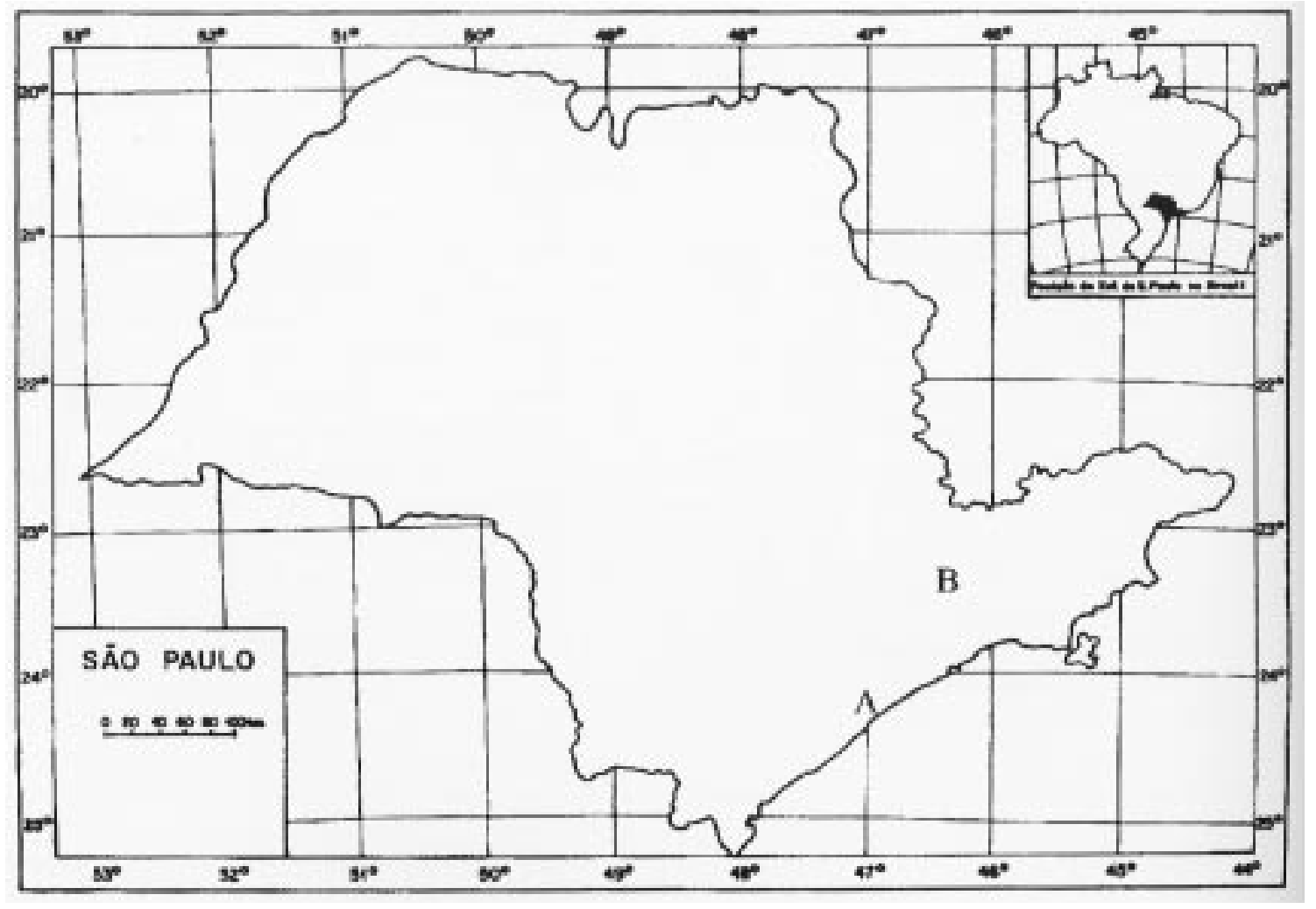

Figura 1. Mapa de localização da Estação Ecológica da Juréia (A) e Parque Estadual da Cantareira (B). 
Tabela 1. Características dos sítios de estudo localizados no Parque Estadual da Cantareira e Estação Ecológica da Juréia.

\begin{tabular}{cccccc}
\hline Sítio & $\begin{array}{c}\text { Elevação } \\
(\mathrm{m})\end{array}$ & $\begin{array}{c}\text { Prec.anual } \\
(\mathrm{mm})\end{array}$ & $\begin{array}{c}\text { Estação } \\
\text { seca* }\end{array}$ & $\begin{array}{c}\text { Clima } \\
\text { (Koeppen) }\end{array}$ & $\begin{array}{c}\text { Solo } \\
\text { tipo }\end{array}$ \\
\hline Cantareira & 900 & 1400 & 4 & Cfb & latossolo \\
Juréia & 300 & 2200 & 0 & Af & podzólico \\
\hline
\end{tabular}

* meses < 60 mm de precipitação.

As espécies foram classificadas com base na estrutura demográfica de suas populações nos locais de estudo (Mantovani 1993, Tabarelli 1994) e em informações sobre suas biologias publicadas em Reitz (1965/1989).

Através do teste $t$ (Zar 1984), comparou-se a abundância relativa média de indivíduos das diferentes guildas de regeneração observadas nas clareiras naturais. Utilizando-se teste de regressão linear (Zar 1984), analisou-se a relação entre o tamanho das clareiras e o número de indivíduos amostrados, o número de espécies, a abundância relativa de indivíduos e espécies das guildas de regeneração e a abundância das espécies dominantes.

\section{Resultados}

Colonização das clareiras - Na floresta na Cantareira foram amostradas clareiras com tamanhos entre $34,9 \mathrm{~m}^{2}$ e $117 \mathrm{~m}^{2}$ (tabela 2). Não encontrou-se correlação significativa entre as variáveis: tamanho das clareiras, abundância de indivíduos e de espécies das diferentes guildas e abundância das espécies dominantes. Encontrou-se correlação significativa entre o tamanho das clareiras e o número de indivíduos e de espécies amostradas $(\mathrm{r}=0,97, \mathrm{~F}=122,9, \mathrm{P}<0,05$; $\mathrm{r}=0,81, \mathrm{~F}=13,47, \mathrm{P}<0,05)$.

$\mathrm{Na}$ Juréia amostraram-se clareiras entre $51,8 \mathrm{~m}^{2}$ e $256,3 \mathrm{~m}^{2}$, onde apenas 3 apresentaram áreas superiores a $150 \mathrm{~m}^{2}$, totalizando $34,17 \%$ da área total ocupada por clareiras nesta floresta (tabela 3 ).

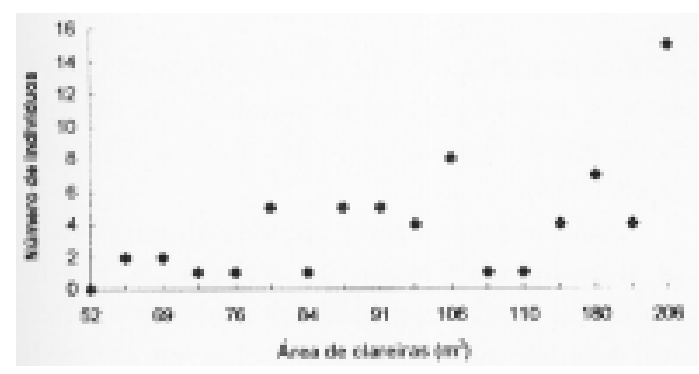

Figura 2. Abundância de Euterpe edulis em clareiras de diferentes tamanhos na floresta da Juréia, SP.
Não encontrou-se correlação entre o tamanho das clareiras, a abundância de indivíduos e de espécies das diferentes guildas e a abundância de Cecropia glaziovii, Euterpe edulis e Chrysophyllum flexuosum, as espécies mais abundantes observadas nas clareiras desta floresta. A abundância de Euterpe edulis, nas clareiras de diferentes tamanhos, exemplifica a relação observada (figura 2).

Encontrou-se correlação significativa entre o tamanho das clareiras e o número de indivíduos e de espécies amostradas $(\mathrm{r}=0,76, \mathrm{~F}=49,17, \mathrm{P}<0,05$; $\mathrm{r}=0,65, \mathrm{~F}=28,76, \mathrm{P}<0,05)$.

No conjunto das clareiras amostradas na floresta da Cantareira e Juréia, 20 (86,9\%) são pequenas e três $(13,1 \%)$ são grandes (figura 3).

Riqueza e abundância das espécies - Na análise das clareiras naturais na Cantareira, foram amostrados 304 indivíduos pertencentes a 64 espécies arbóreo-arbustivas, onde apenas oito $(12,5 \%)$ são intolerantes à sombra (figura 4). Na Juréia foram amostrados 254 indivíduos pertencentes a 71 espécies arbóreo-arbustivas, sendo sete $(9,85 \%)$, intolerantes à sombra (tabela 5).

Nas clareiras nas duas florestas, as espécies intolerantes à sombra foram pouco frequientes e pouco abundandes (tabela 4).

Na floresta da Cantareira e Juréia, a abundância relativa de indivíduos das espécies tolerantes à sombra é significativamente maior que a abundância relativa de indivíduos das intolerantes à sombra $(\mathrm{t}=41,8, \mathrm{~g} .1 .=10, \mathrm{P}<0,001 ; \mathrm{t}=17.5, \mathrm{~g} .1 .=32, \mathrm{P}<$ 0.001 ) (tabelas 2 e 3 ).

Os resultados encontrados foram contrários às predições iniciais do estudo.

\section{Discussão}

Colonização das clareiras - Estudos em algumas florestas tropicais (Uhl \& Murphy 1981, Brokaw 1985, Arriaga 1988, Whitmore 1989) indicaram que clareiras pequenas são mais freqüentes do que as grandes. No entanto clareiras grandes são 
Tabela 2. Clareiras naturais com suas respectivas áreas (A), número de espécies encontradas (B), densidade de indivíduos (C), indivíduos intolerantes à sombra (D), indivíduos tolerantes à sombra de dossel (E) e indivíduos tolerantes à sombra de sub-bosque (F), amostrados na floresta da Cantareira, SP*.

\begin{tabular}{ccccccc}
\hline clareira & $\mathrm{A}\left(\mathrm{m}^{2}\right)$ & $\mathrm{B}$ & $\mathrm{C}\left(\mathrm{m}^{2}\right)$ & $\mathrm{D} \% * *$ & $\mathrm{E} \% * *$ & $\mathrm{~F} \% * *$ \\
\hline 1 & 34,9 & 17 & 1,11 & 5,1 & 46,1 & 48,7 \\
2 & 29,1 & 13 & 0,99 & 3,4 & 51,7 & 44,8 \\
3 & 48,4 & 15 & 0,99 & 4,1 & 43,7 & 52,0 \\
4 & 70,0 & 31 & 0,91 & 10,2 & 51,2 & 38,4 \\
5 & 49,0 & 21 & 0,95 & 3,0 & 58,4 & 38,4 \\
6 & 117,0 & 21 & 0,67 & 11,5 & 38,2 & 50,1 \\
\hline Total & 348,5 & & $\bar{x} \pm$ D.P. & $6,2 \pm 3,6$ & $48,2 \pm 7,0$ & $45,4 \pm 5,9$ \\
\hline
\end{tabular}

* indivíduos entre $0,5 \mathrm{~m}$ e $1,3 \mathrm{~m}$. ** abundância relativa.

Tabela 3. Clareiras naturais com suas respectivas áreas (A), número de espécies encontradas (B), densidade de indivíduos (C), indivíduos intolerantes à sombra (D), indivíduos tolerantes à sombra de dossel (E) e indivíduos tolerantes à sombra de sub-bosque (F), amostrados na floresta da Juréia, SP*.

\begin{tabular}{|c|c|c|c|c|c|c|}
\hline clareira & $A\left(m^{2}\right)$ & B & $\mathrm{C}\left(\mathrm{m}^{2}\right)$ & $\mathrm{D} \% * *$ & $\mathrm{E} \% * *$ & $\mathrm{~F} \% * *$ \\
\hline 1 & 179,8 & 11 & 0,10 & 5,5 & 16,6 & 77,7 \\
\hline 2 & 80,3 & 13 & 0,21 & 5,2 & 36,8 & 57,8 \\
\hline 3 & 256,3 & 21 & 0,15 & 5,0 & 20,0 & 75,0 \\
\hline 4 & 106,0 & 4 & 0,10 & 0,0 & 0,0 & 100,0 \\
\hline 5 & 104,6 & 9 & 0,15 & 0,0 & 31,2 & 68,7 \\
\hline 6 & 198,7 & 21 & 0,13 & 0,0 & 26,9 & 73,3 \\
\hline 7 & 84,8 & 10 & 0,20 & 0,0 & 11,7 & 88,2 \\
\hline 8 & 106,8 & 7 & 0,12 & 46,1 & 0,0 & 53,8 \\
\hline 9 & 51,8 & 6 & 0,15 & 12,5 & 12,5 & 75,0 \\
\hline 10 & 120,4 & 7 & 0,12 & 40,0 & 13,3 & 46,6 \\
\hline 11 & 109,6 & 12 & 0,19 & 0,0 & 4,7 & 95,2 \\
\hline 12 & 76,3 & 5 & 0,06 & 0,0 & 33,3 & 66,6 \\
\hline 13 & 72,2 & 8 & 0,12 & 0,0 & 0,0 & 100,0 \\
\hline 14 & 84,2 & 7 & 0,08 & 14,2 & 28,5 & 57,1 \\
\hline 15 & 66,3 & 7 & 0,10 & 0,0 & 12,5 & 8,5 \\
\hline 16 & 68,6 & 6 & 0,13 & 0,0 & 22,2 & 77,7 \\
\hline 17 & 90,7 & 6 & 0,12 & 0,0 & 0,0 & 100,0 \\
\hline Total & 1856,1 & & $\overline{\mathrm{X}} \pm$ D.P. & $7,5 \pm 14,1$ & $15,9 \pm 12,4$ & $76,4 \pm 16,9$ \\
\hline
\end{tabular}

* indivíduos com $(\mathrm{PAP})>5 \mathrm{~cm}$. ** abundância relativa.

responsáveis pela maior parte da superfície coberta por este tipo de perturbação (Martinez-Ramos 1985). Os resultados encontrados sugerem que na Cantareira e na Juréia, clareiras pequenas são mais freqüentes e contêm a maior parte da superfície coberta por este tipo de distúrbio (figura 3).

O tamanho das clareiras naturais causadas por morte e queda de árvores está relacionado ao número e ao porte dos indivíduos envolvidos. Uma clareira natural pode originar-se da morte de uma árvore do dossel da floresta que se mantém em pé, criando uma clareira pequena, ou originar-se pela queda de várias árvores, criando clareiras grandes, que em alguns casos apresentam áreas superiores a 70 hectares (Web 1958).

A não observação de grandes clareiras naturais na floresta na Cantareira (tabela 2), pode ser explicada, em parte, pela altura média desta floresta, seu estádio sucessional e pelas características climáticas e fisiográficas locais (tabela 1).

Trata-se de uma floresta secundária tardia (100-120 anos) (Tabarelli 1994), com dossel 


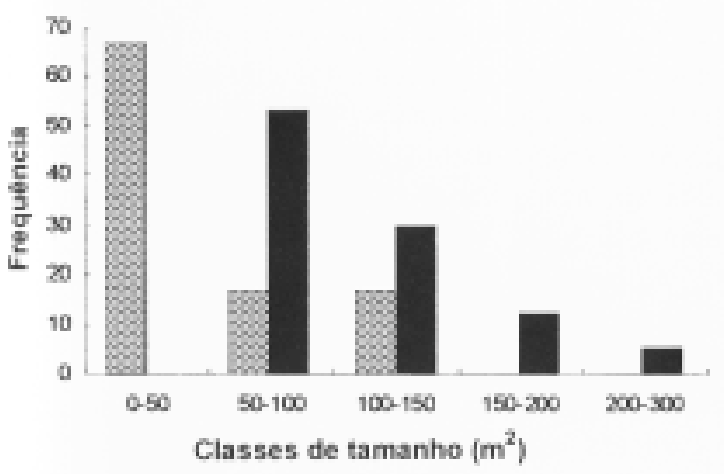

Figura 3. Freqüência de clareiras por classes de tamanho nas florestas da Cantareira ( $)$ ) e Juréia ( $\square$ ), SP.

constituído por árvores com alturas médias entre $20 \mathrm{~m}$ e $25 \mathrm{~m}$, que ao caírem não são capazes de abrirem grandes clareiras. A morte de indivíduos de espécies pioneiras e secundárias, as quais são muito abundantes, favorece a ocorrência de muitos indivíduos mortos em pé, originando número elevado de clareiras pequenas. São raras as grandes tempestades que ocasionam a queda múltipla de árvores, mesmo ocorrendo chuvas intensas no período chuvoso.

A Estação Ecológica da Juréia é um local de relevo acidentado, coberto por florestas com alturas entre $15 \mathrm{~m}$ e $30 \mathrm{~m}$. No período chuvoso ocorrem precipitações elevadas, mas também são raras as tempestades tropicais. Muitos trechos destas florestas estão abrigados dos ventos fortes como nos fundos de vales e encostas expostas para o continente. Estes fatores, aliados à amostragem pequena, podem ter contribuído para a raridade de grandes clareiras observadas (tabela 3 ).

Dentro do espectro de tamanho de clareiras analisadas, não se encontrou relação entre o tamanho das mesmas, a abundância relativa das guildas e a abundância das espécies dominantes, como é o caso de Euterpe edulis na floresta da Juréia (figura 2).

Estes resultados podem ser conseqüência da intensidade do distúrbio observado nas clareiras, da amplitude de nicho de regeneração das espécies e/ou refletir diferenças de composição florística entre fisionomias florestais, as quais circundam as clareiras amostradas. A ocorrência de diferentes fisionomias está relacionda às condições fisiográficas locais (Mantovani 1990).

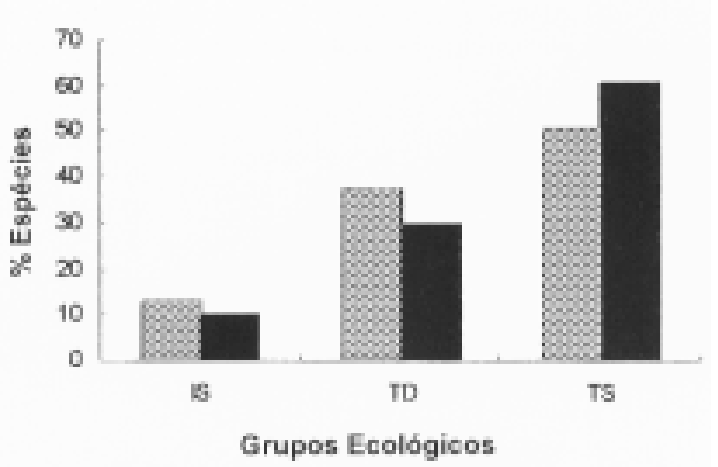

Figura 4. Abundância relativa de espécies intolerantes à sombra (IS), tolerantes à sombra de dossel (TD) e tolerantes à sombra de sub-bosque (TS), amostradas nas clareiras nas florestas da Cantareira ( $\because$ ) e Juréia ( $\square$ ), SP.

Brokaw (1985) analisou 30 clareiras naturais em Barro Colorado, Panamá, encontrando uma relação significativa entre a densidade de indivíduos das espécies intolerantes à sombra e a área das clareiras quando estas apresentavam entre 5 e 6 anos de idade. Não encontrou a mesma relação para as espécies tolerantes à sombra.

Este autor acredita que estes resultados estão de acordo com a predição de Connell \& Slatyer (1977), segundo os quais as espécies primárias (tolerantes à sombra) podem regenerar em clareiras grandes e pequenas, desde que o distúrbio (alteração ambiental) não seja muito severo. Este pode ser o caso das grandes clareiras que, em função de suas localizações, nunca recebem luz solar direta em seus interiores, apresentando condições ambientais similares às observadas nas pequenas.

Evidência para esta hipótese, é o comportamento de algumas espécies de Chusquea (bambu), que ocorrem em pequenas e grandes clareiras nas florestas de topos de morro, mas somente em grandes nas dos fundos de vale.

Outra hipótese é que a maioria das árvores tropicais apresenta nicho amplo de regeneração (Hubbell \& Foster 1987), o que determina baixa previsibilidade da composição de espécies e guildas de regeneração das clareiras naturais.

$\mathrm{Na}$ floresta Atlântica muitas espécies de sub-bosque ocorrem em florestas secundárias de diferentes idades e em florestas maduras com diferentes estruturas, sugerindo nicho amplo de regeneração. Como exemplo, podemos citar Rudgea jasminoides, Guatteria nigrescens e Mollinedia 
Tabela 4. Freqüência e abundância das espécies intolerantes à sombra observadas em clareiras naturais nas florestas da Cantareira e Juréia, SP.

\begin{tabular}{lcc}
\hline $\begin{array}{l}\text { Local } \\
\text { Espécie }\end{array}$ & $\begin{array}{c}\text { Freqüência } \\
\text { (no de clareiras) }\end{array}$ & $\begin{array}{c}\text { № total de } \\
\text { indivíduos } \\
\text { amostrados }\end{array}$ \\
\hline Cantareira & & \\
$\quad$ Alchornea triplinervia & 2 & 2 \\
$\quad$ Croton floribundus & 1 & 1 \\
Dalbergia frutescens & 1 & 1 \\
$\quad$ Inga marginata & 3 & 3 \\
Leandra moseni & 2 & 2 \\
Miconia cabucu & 1 & 2 \\
$\quad$ Miconia latecrenata & 2 & 5 \\
Piptadenia gonoachanta & 1 & 1 \\
$\quad$ & & \\
Juréia & & 13 \\
Cecropia glaziovii & 4 & 2 \\
Erioteca pentaphyla & 2 & 2 \\
Ilex theezans & 2 & 2 \\
Inga affinis & 2 & 1 \\
Inga marginata & 1 & 1 \\
Miconia pyrifolia & 1 & \\
Pera glabrata & 1 & \\
\hline
\end{tabular}

schottiana. Comportamento semelhante, porém restrito às florestas secundárias, apresentam algumas grandes pioneiras como Alchornea triplinervia e Cedrela fissilis.

Relativo às diferenças de composição florística, a floresta Atlântica é um mosaico de tipos florestais, conseqüência das variações de relevo, micro-clima e tipos de solo, entre outros fatores (Mantovani 1990, Mantovani et al. 1990). A colonização das clareiras naturais, provavelmente, reflete a heterogeneidade florística da comunidade, associada às condições fisiográficas locais, o que dificulta estabelecer relações entre o tamanho da clareiras e os padrões de colonização, principalmente no que refere-se a abundância das espécies.

Como exemplo, pode-se citar Euterpe edulis, espécie que ocorre em clareiras somente nas florestas nos fundos de vales e naquelas nas médias-encostas. A análise estratificada por tipo de vegetação, exposição solar e condição topográfica (topo de morro, média-encosta, etc.) surge como um pré-requisito para o estudo das relações entre a estrutura e a colonização de clareiras.
No caso de clareiras ocupadas, predominantemente, por espécies tolerantes à sombra, o padrão de colonização pode refletir também a dinâmica do sub-bosque da floresta anterior a abertura das mesmas, o que dificulta prever suas composições.

A correlação positiva observada, entre o tamanho das clareiras e o número de indivíduos e espécies amostradas, (tabelas 2 e 3 ) sugere que independente de suas origens e tamanhos, as clareiras apresentam o mesmo potencial de colonização e/ou refletem o fato de serem ocupadas por indivíduos previamente estabelecidos. Esta é uma evidência favorável à hipótese de que grandes clareiras, em deteminadas condições, funcionam como pequenas.

Os resultados encontrados em outras florestas tropicais não fornecem as mesmas evidências. Em La Selva, por exemplo, mais de $80 \%$ dos indivíduos amostrados nas clareiras se estabeleceram posteriormente às suas aberturas (Brandani et al. 1988).

Riqueza e abundância das espécies - A contribuição das clareiras à riqueza da comunidade relaciona-se, entre outros aspectos, ao número de espécies que dependem efetivamente destes sítios, para obterem uma regeneração exitosa, como é o caso das espécies intolerantes à sombra.

As clareiras naturais observadas em ambas florestas, apresentaram um padrão comum de colonização, caracterizado por abundância e riqueza reduzida de indivíduos e espécies intolerantes à sombra, as quais são também pouco freqüentes (tabelas 2, 3 e 4).

Costa \& Mantovani (1992) obtiveram resultados similares ao analisarem 21 clareiras $\left(10-180 \mathrm{~m}^{2}\right) \mathrm{em}$ um fragmento de floresta Atlântica. Foram amostradas 69 espécies de árvores, das quais somente $15(21,7 \%)$ são intolerantes à sombra. Ao contrário destes estudos, Brokaw (1985) analisou 30 clareiras naturais $\left(20-705 \mathrm{~m}^{2}\right)$ na Ilha de Barro Colorado, onde amostrou 90 espécies, das quais 37 $(41,1 \%)$ são intolerantes à sombra.

Entre os fatores que podem explicar parte dos resultados obtidos nas florestas da Cantareira e Juréia (figura 4), destacam-se o regime de perturbação natural das florestas, suas condições fisiográficas e, a amostragem de arbustos e pequenas árvores. 
Tabela 5. Espécies amostradas nas clareiras naturais nas florestas da Cantareira e da Juréia, SP.

\begin{tabular}{|c|c|}
\hline $\begin{array}{l}\text { Guildas de regeneração } \\
\text { Espécies }\end{array}$ & $\begin{array}{l}\text { Guildas de regeneração } \\
\text { Espécies }\end{array}$ \\
\hline Intolerantes à sombra & Chrysophyllum flexuosum Mart. \\
\hline Alchornea triplinervia (Spreng.) Müll. Arg. & Citronella megaphylla (Miers) Howard \\
\hline Cecropia glaziovii Sneth. & Coccoloba velloziana Casar \\
\hline Clethra scabra Pers. & Cordia sellowiana Cham. \\
\hline Croton floribundus Sreng. & Dahlstedtia pinnata (Benth.) Malme \\
\hline Dalbergia frutescens (Vell.) Britton & Daphnopsis geminiflora (Miers) Domcke \\
\hline Eriotheca pentaphyla (Vell.) K. Schum & Diclidanthera laurifolia Mart. \\
\hline Ilex theezans Mart. & Ecclinusa ramiflora Mart. \\
\hline Inga affinis DC. & Erythroxylum cuspidatum Mart. \\
\hline Inga marginata Willd. & Eugenia bocainensis Mattos \\
\hline Miconia latecrenata Naud. & Eugenia cuprea Berg \\
\hline Miconia pyrifolia Naud. & Eugenia flavecens DC. \\
\hline Pera glabrata Baill. & Eugenia glomerata Spreng. \\
\hline Piptadenia gonoacantha (Mart.) Macbride & Eugenia oblongata Berg \\
\hline Vernonia discolor Less. & Eugenia obovata Berg \\
\hline Tolerantes à sombra de dossel & Eugenia riedeliana Berg \\
\hline Cariniana estrellensis (Raddi) O. Ktze. & Eugenia umbelliflora DC. \\
\hline Casearia decandra Jacq. & Euterpe edulis Mart. \\
\hline Casearia sylvestris Sw. & Faramea montevidensis (Cham. et Schl.) DC. \\
\hline Chrysophyllum inornatum Mart. & Gomidesia spectabilis (DC.) Berg \\
\hline Chrysophyllum marginatum (Hook. \& Arn.) Radlk. & Guarea macrophylla Vahl \\
\hline Cordia superba Cham. & Guatteria australis St. Hil. \\
\hline Cryptocaria moschata Mart. et Nees & Guatteria nigrescens Mart. \\
\hline Cupania oblongifolia Camb. & Hirtella hebeclada Moric. \\
\hline Dendropanax cuneatum E. March. & Inga capitata Desv. \\
\hline Endlicheria paniculta (Spreng.) Macbride & Ixora burchelliana Müll. Arg. \\
\hline Ficus insipida Willd. & Leandra mosenii Cogn. \\
\hline Guapira opposita (Vell.) Reitz & Machaerium oblongifolium Vog. \\
\hline Heisteria silvianii Schwacke & Marliera obscura Berg \\
\hline Licania hoehnei Pilg. & Marliera suaveolens Camb. \\
\hline Licania octandra Pilg. & Marliera tomentosa Camb. \\
\hline Matayba elaeagnoides Radlk. & Metrodora nigra St. Hil. \\
\hline Matayba guianensis Aubl. & Mollinedia schottiana (Spreng.) Perk. \\
\hline Myrcia rostrata DC. & Mollinedia uleana Perk. \\
\hline Nectandra leucantha Nees et Mart. & Myrceugenia kleinii Legr. et Kraus \\
\hline Nectandra saligna Nees et Mart. & Myrcia aff laruoteana Berg \\
\hline Ocotea diospyrifolia (Meiss.) Mez & Myrcia leptoclada DC. \\
\hline Ocotea dispersa (Meiss.) Mez & Myrcia multiflora (Lam.) DC. \\
\hline Ocotea divaricata (Nees) $\mathrm{Mez}$ & Neomitranthes glomerata (Legr.) Legr. \\
\hline Ocotea odorifera (Vell.) Roher & Neuraputia saldanhense Emmerich \\
\hline Ocotea silvestris Vatt. & Ouratea parviflora (DC.) Baill. \\
\hline Pouteria laurifolia (Gomes) Radlk. & Pilocarpus pauciflorus St. Hil. \\
\hline Rollinia sericea R. E. Fries & Piper aduncum $\mathrm{L}$. \\
\hline Sloanea guianensis Aubl. & Piper amalago L. \\
\hline Swartzia langsdorffii Raddi & Posoqueria acutifolia Mart. \\
\hline Virola oleifera (Schott) A.C. Smith & Psychotria capitata DC. \\
\hline Xylopia brasiliensis Spreng. & Psychotria cephalantus (Müll. Arg.) Standl. \\
\hline Xylopia langsdorfiana St. Hil. \& Tul. & Psychotria kleinii Smith. \& Downs \\
\hline Tolerantes à sombra de sub-bosque & Psychotria pubigera Schlecht. \\
\hline Alibertia myrcifolia Schum. & Psychotria suterella Müll. Arg. \\
\hline Amphirrox longifolia Spreng. & Pterocarpus rohrii Vahl. \\
\hline Andira anthelmia (Vell.) Macbride & Rheedia gardneriana Planch. et Triana \\
\hline Bactris setosa Mart. & Rudgea jasminoides (Cham.) Müll. Arg. \\
\hline Calycorectes australis Legr. & Rustia formosa (Cham. \& Schlecht.) Kl. \\
\hline Calyptranthes concina DC. & Seguieria guaranitica Speg. \\
\hline Calyptranthes grandifolia Berg & Siphoneugena densiflora Berg \\
\hline Calyptranthes lanceolata Berg & Sorocea bonplandii (Baill.) W. Burg. \\
\hline Calyptranthes lucida Mart. ex DC. & Urera nitida (Vell.) Brack \\
\hline Cestrum schlechtendalii D. Don & Xylosma glaberrimun Sleumer \\
\hline
\end{tabular}


Whitmore (1989) previu que, em florestas onde predominam clareiras pequenas, a riqueza de espécies intolerantes à sombra é reduzida. Segundo Brokaw (1985), espécies pioneiras são mais freqüentes em clareiras maiores do que $200 \mathrm{~m}^{2}$. Os resultados deste estudo sugerem que as clareiras naturais pequenas são mais abundantes do que as grandes e ocupam a maior parte da área coberta por este tipo de distúrbio (figura 3).

Ao contrário das florestas tropicais estabelecidas sobre planícies, a floresta atlântica situa-se sob relevo montanhoso. Clareiras em locais com exposição solar SE e SW jamais recebem luz solar direta em seus interiores, o que restringe o estabelecimento de espécies intolerantes à sombra. Neste caso, grandes clareiras podem funcionar ecologicamente como pequenas, o que diminui a heterogeneidade ambiental.

A inclusão de arbustos e pequenas árvores na análise de colonização influenciou a riqueza e a abundância de indivíduos de espécies tolerantes à sombra amostradas, das quais, grande parte, é típica de sub-bosque (figura 4). Em média, 45,4\% e 76,4\% dos indivíduos amostrados nas clareiras pertencem às espécies de sub-bosque (tabelas 2 e 3). Destacam-se espécies de Myrtaceae, Melastomataceae, Rubiaceae, Piperaceae e Monimiaceae (Klein 1980, Silva \& Leitão-Filho 1982, Mantovani et al. 1990).

Estudos em várias florestas neotropicais (Gentry 1986, Hamell 1990, Hubbel1 \& Foster 1990, Prance 1990, Melo 1994) têm demonstrado a riqueza elevada deste grupo de plantas. Arbustos e pequenas árvores são comumente desprezados nos estudos de colonização de clareiras, subestimando-se a importância das espécies tolerantes à sombra na ocupação destes ambientes, enfatizando a ocorrência das intolerantes.

Na floresta atlântica do sul e sudeste do Brasil não existem estimativas sobre a diversidade e a contribuição das espécies intolerantes à sombra para a riqueza da comunidade. Observam-se espécies pioneiras (Budowski 1965), que ocorrem associadas à sucessão secundária em áreas submetidas a ação antrópica severa. Destacam-se os gêneros Vernonia, Baccharis e Piptocarpha (Klein 1980, Feitosa do Nascimento 1994, Knobel 1995). Em florestas livres de intervenção antrópica, estas espécies devem ocorrer em grandes clareiras ou em locais dominados por vegetação arbustiva como nos topos dos morros.
Podem também serem oriundas da floresta estacional que, em algumas regiões, circunda a floresta atlântica. A atividade humana resulta freqüentemente na expansão da vegetação ruderal sobre a floresta perturbada (Ewel 1980). A riqueza deste grupo está associada aos níveis de intervenção humana (Gomez-Pompa 1971).

Encontram-se também as grandes pioneiras (Swaine \& Whitmore 1988) ou secundárias (Budowski 1965), muito abundantes nas florestas perturbadas ou sucessionais. Nas florestas livres de perturbação antrópica se estabelecem em clareiras naturais e na fase de reconstrução (Tabarelli 1994) Destacam-se as espécies de Alchornea, Pera, Croton e Piptadenia, entre outras. Ocorrem também as pioneiras de ciclo de vida curto, como espécies de Cecropia e Tibouchina (Klein 1980, Smith et al. 1988, Pompéia 1990, Tabarelli et al. 1994).

Entre as espécies intolerantes à sombra observadas neste estudo (tabela 4), Inga marginata, Inga affinis e Dalbergia frutescens ocorrem frequientemente nas bordas das florestas ao longo de cursos d'água (Burkart 1979). Ilex theezans, Miconia pyrifolia e Miconia latecrenata ocorrem em florestas abertas em topos de morros, como a floresta nebular (Falkenberg \& Voltolini 1993), onde regeneram sob o dossel (Klein 1980). Vernonia discolor e Clethra scabra são espécies ruderais, associadas à sucessão secundária em florestas que sofreram perturbações antrópicas severas. Somente Cecropia glaziovii e Piptadenia gonoachanta podem apresentar dependência efetiva das clareiras.

Os resultados deste estudo sugerem que, na floresta atlântica no estado de São Paulo, as clareiras naturais pequenas são mais abundantes do que as grandes e ocupam a maior parte da área coberta por este tipo de distúrbio. As espécies não apresentam partição de nicho regenerativo em relação ao tamanho das clareiras, o qual não é o melhor preditor da composição florística e ecológica das mesmas.

Esta floresta apresenta um número reduzido de espécies intolerantes à sombra, principalmente do grupo das grandes pioneiras. São espécies pouco freqüentes e pouco abundantes, sendo que muitas destas parecem regenerar com sucesso em outros hábitats, como nas florestas abertas sobre solos rasos, fato que diminui a importância das clareiras naturais, como mecanismo de manutenção da riqueza deste grupo de espécies. 
A abundância reduzida de grandes clareiras pode ser compensada pela diversidade da paisagem, o que proporciona às clareiras de mesmo tamanho, condições, distintas, criando a heterogeneidade ambiental necessária à especialização (Tilman 1985).

Florestas estabelecidas sobre condições fisiográficas semelhantes devem apresentar características similares às observadas neste estudo.

A amostragem reduzida, o pequeno número de grandes clareiras observadas e a baixa freqüência com que ocorreram as espécies não permitem conclusões definitivas sobre as hipóteses testadas neste estudo. Novos estudos, incluindo um maior número de clareiras dentro de amostragens estratificadas por fisionomias florestais, são necessários para a obtenção de conclusões seguras.

\section{Referências bibliográficas}

ARRIAGA, L. 1988. Gap dynamics of a tropical cloud forest in Northeasten México. Biotropica 20:178-184.

BAZZAZ, F.A. \& PICKETT, S.T.A. 1980. Physiological ecology of tropical succession: a comparative review. Annu. Rev. Ecol. Syst. 11:287-310.

BEGON, M., HARPER, J.L. \& TOWNSEND, C.R 1990 Ecology: individuals, populations and communities Blackwell, Oxford.

BERG, C.C. 1978. Espécies de Cecropia da Amazônia Brasileira. Acta Amaz. 8:149-182.

BRANDANI, A., HARTSHORN, G.S. \& ORIANS, G.H. 1988. Internal heterogeneity of gaps and species richness in Costa Rica tropical wet forest. J. Trop. Ecol. 68:99-119

BROKAW, N.V.L. 1982. The definition of treefall gap and its effects on measures of forest dynamics. Biotropica 11:158-160.

BROKAW, N.V.L. 1985. Gap-phase regeneration in a tropical forest. Ecology 66:682-687.

BUDOWSKI, G. 1965. Distribution of tropical American rain forest species in the light of successional process. Turrialba 15:40-2.

BURKART, A. 1979. Leguminosas. Fl. Ilus. Catar. 1:1-299.

CONNELL, J.H. \& SLATYER, R.O. 1977. Mechanisms of succession in natutal communities and their role in community stability and organization. Am. Nat. 111:1119-1144

COSTA, M \& MANTOVANI, W. 1992. Composição e estrutura de clareiras em mata mesófila na Bacia de São Paulo. In II Congresso nacional de essências nativas, São Paulo, SP. Revta Inst. Ftal 1:178-173.

DENSLOW, J. S. 1980. Gap partioning among tropical rain forest trees. Biotropica 12:(suppl.) 47-55.

DENSLOW, J.S. 1985. Disturbance - mediated coexistence of species. In The ecology of natural disturbance and patch dynamics (S.T.A. Pickett \& P.S White, eds.). Academic Press Inc., New York, p.307-322.

DENSLOW, J.S. 1988. Tropical rain forest gaps and tree species diversity. Annu. Rev. Ecol. Syst. 18:431-451.

EWEL, J. 1980. Tropical succession: manifold routes to maturity. Biotropica 12:(suppl) 2-7.
FALKENBERG, D.B. \& VOLTOLINI, J.C. 1993. The montane cloud forest in the Southern Brazil. In Proceendings of tropical montane cloud forest symposium. Porto Rico, p.86-93.

FEITOSA DO NASCIMENTO, F.A. 1994. A sucessão secundária inicial na mata atlântica, sobre a Serra de Paranapiacaba, Ribeirão Grande, SP. Dissertação de mestrado, Universidade de São Paulo, São Paulo.

GENTRY, A. H. 1986. Endemism in tropical versus temperate plant communities. In Conservation biology, the science of scarcity and diversity (M. Soulé, ed.). Sinauer Associates Inc., Suderland, p.153-181.

GOMEZ-POMPA, A. 1971. Posible papel de la vegetación secundária en la evolución de la flora neotropical Biotropica 3:125-135.

HAMMEL, B. 1990. The distribution of diversity among families, genera and habitat types in the La Selva flora. In Four neotropical rain forests (A.H. Gentry, ed.). Yale University Press, London, p.75-84.

HARTSHORN, G.S. 1978. Treefalls and tropical forest dynamics. In Tropical trees as living systems (P.B. Tomlinson \& M.H Zimmermman, eds.). Cambridge Univ. Press, New York, p.617-638.

HARTSHORN, G.S. 1980. Neotropical forest dynamics. Biotropica 12:(suppl.) 23-30.

HUBBELL, S.P. \& FOSTER, R.G. 1983. Diversity of canopy trees in a neotropical forest and implications for conservation. In Tropical rain forest: ecology and manegement (S.L. Sutton, ed.). Blackwell Sci. Publ., Oxford, p. 25-41.

HUBBELL, S.P. \& FOSTER, R.G. 1986. Canopy gaps and the dynamics of a neotropica1 rain forest. In Plant ecology (R.J. Crawley, ed.). Blackwell Scientific, Oxford, p.77-96.

HUBBELL, S.P. \& FOSTER, R.G. 1987. The spatial context of regeneration in a neotropical forest. In Colonization, succession and stability (R.J. Crawley, ed.). Blackwell, London, p.395-414.

HUBBELL, S.P. \& FOSTER, R.B. 1990. The floristic composition of the Barro Colorado island forest. In Four neotropical rain forests (A.H. Gentry, ed.). Yale University Press, London, p.85-98.

KLEIN, R.M. 1980. Ecologia da flora e vegetação do Vale do Itajaí. Sellowia 32:165-389.

KNOBEL, M.G. 1995. Aspectos da regeneração natural do componente arbóreo-arbustivo, de trecho da floresta da Reserva Biológica do Instituto de Botânica em São Paulo, SP. Dissertação de mestrado, Universidade de São Paulo, São Paulo.

MANTOVANI, W. 1990. A dinâmica da floresta na encosta atlântica. In II Simpósio ecossistemas costa sul e sudeste brasileira: estrutura, função e manejo, vol. 1 (ACIESP, ed.), São Paulo, p.304-13.

MANTOVANI, W. 1993. Estrutura e dinâmica da floresta Atlântica na Juréia, Iguape-SP. Tese de livre-docência, Universidade de São Paulo, São Paulo.

MANTOVANI, W., RODRIGUES, R.R., ROSSI, L., ROMANIUC-NETO, S., CATHARINO, E.L.M. \& CORDEIRO, I. 1990. A vegetação na Serra do Mar em Salesópolis. In II Simpósio ecossistemas da costa sul e sudeste: estrutura composição e manejo, vol. 2 (ACIESP, ed.), São Paulo, p.348-384.

MARTINEZ-RAMOS, M. 1985. Claros, ciclos vitales de árboles tropicales y regeneración natural de las selvas altas perennifolias. In Investigaciones sobre la regeneración de selvas altas en Veracruz, México (A. Gómez-Pompa \& S. del Amo, eds.). Editorial Alhambra Mexicana, México, p.191-239. 
MELO, M. R. F. 1994. Composição florística e estrutura fitossociológica da mata atlântica de encosta na Ilha do Cardoso (Cananéia, Brasil). Bol. Inst. Bot. 9:107-158.

ORIANS, G. H. 1982. The influence of tree-falls in tropical forest in tree species richness. Trop. Ecol. 23:255-279.

PRANCE, G.T. 1990. The floristic composition of the forests of Central Amazonian. In Four neotropical rain forests (A.H. Gentry, ed.). Yale University Press, London, p.112-140.

POMPÉIA, S.L. 1990. Recuperação do ecossistema mata atlântica de encosta. In VI Congresso florestal brasileiro, vol. 1 (S.B.S., ed.), São Paulo, p.147-155

REITZ, R. 1965/1989. Flora Ilustrada Catarinense. Herbário Barbosa Rodrigues. Itajaí, SC. 149 vol.

SILVA, A.F. \& LEITÃO-FILHO, H.F. 1982. Composição florístíca e estrutura de um trecho da mata atlântica de encosta no Município de Ubatuba (São Paulo, Brasil). Revta brasil. Bot. 5:43-52.

SMITH, L.B., DOWNS, R.J. \& KLEIN, R.M. 1988. Euforbiáceas. Flora Ilustrada Catarinense 1:1-408.

SWAINE, M. D. \& WHITMORE, T. C. 1988. On the definition of ecological species groups in tropical rain forest. Vegetatio 75:81-86.

TABARELLI, M. 1994. Clareiras naturais e a dinâmica sucessional de um trecho de floresta secundária na Serra da Cantareira, SP. Tese de mestrado, Universidade de São Paulo, São Paulo.

TABARELLI, M., VILLANI, J.P \& MANTOVANI, W. 1994. Estudo comparativo da vegetação de dois trechos de floresta secundária no Núcleo Santa Virgínia, SP. Revta Inst. Ftal 6:1-11.
TERBORGH, J. 1992. Diversity and the tropical rain forest. Scientific American Library, New York.

TILMAN, D. 1985. The resource-ratio hypothesis of plant succession. Am. Nat. 125:827-852.

UHL, C. \& MURPHY, P. G. 1981. Composition, structure and regeneration of tierra firme forest in the Venezuelan amaron basin. Trop. Ecol. 22:219-236.

VELOSO, H. P., RANGEL FILHO, A. L. R. \& LIMA, J. C. A 1991. Classificação da vegetação brasileira, adaptada a um sistema universal. IBGE, Rio de Janeiro.

VÁZQUEZ-YANES, C. \& SADA, S.G. 1985. Caracterización de los grupos ecológicos de árboles de la selva húmeda. In Regeneración de las selvas altas de Vera Cruz. (A. Gomez-Pampa \& S. Del Amo, eds). Editorial Alhambra Mexicana, México, p.67-78.

WEBB, L. J. 1958. Cyclones as an ecological factor in tropical lowland rainforest, North Queenisland. Aust. J. Bot. 7:220-28.

WHITMORE, T.C. 1978. Gaps in the forest canopy. In Tropical trees as living systems (P. B. Tomlinson \& M.H. Zimmermman, eds.). Cambridge Univ. Press, New York, p.639-655.

WHITMORE, T.C. 1982. On pattern and process in forests. In The plant community as a working mechanism (E.I. Newman, ed.). Blackwell Scientific, Oxford, p.45-59.

WHITMORE, T.C. 1989. Canopy gaps and two major groups of forest trees. Ecology 70:536-538.

WHITMORE, T.C. 1990. An introduction to tropical rainforeste. Blackwell, London.

ZAR, J. H. 1984. Biostatistical analysis. Prentice-Hall, London. 